\title{
Memory for empty time intervals in pigeons
}

\author{
DOUGLAS S. GRANT \\ University of Alberta, Edmonton, Alberta, Canada
}

\begin{abstract}
Pigeons were trained to discriminate short $(2 \mathrm{sec})$ and long $(8 \mathrm{sec})$ empty intervals that began each trial. In group consistent, onset of an empty interval was marked by a brief presentation of red keylight, and termination of the interval was marked by a brief presentation of green keylight. In group inconsistent, red and green served equally often as the first and second markers across trials. Testing revealed that, in group consistent, (1) birds were sensitive to the relation between marker color and marker type and (2) presentation of the second marker did not initiate timing a new interval. Testing also revealed a robust choose-long effect at delays longer than the training delay and indifference between the comparisons on no-sample trials. Both of the latter findings differ from those typically obtained when filled intervals are employed. It was concluded that pigeons process filled and empty intervals differently.
\end{abstract}

Many studies have used a symbolic matching-to-sample task to study memory for event duration in pigeons (see Grant, Spetch, \& Kelly, 1997, for a review). In a typical experiment, trials begin with the presentation of a houselight or keylight stimulus for either a short (e.g., $2 \mathrm{sec}$ ) or a long (e.g., $8 \mathrm{sec}$ ) duration. Following termination of the duration sample, two comparison stimuli are presented for a choice. Choice of one comparison is reinforced on trials initiated by the short sample, and choice of the alternative comparison is reinforced on trials initiated by the long sample.

When subsequently tested at delays longer than the training delay, pigeons demonstrate a choose-short effect (e.g., Grant \& Kelly, 1996, 1998; Grant \& Spetch, 1991, 1993, 1994; Kraemer, Mazmanian, \& Roberts, 1985; Santi, Bridson, \& Ducharme, 1993; Spetch, 1987; Spetch \& Rusak, 1992a; Spetch \& Wilkie, 1982, 1983; Wilkie, 1987). That is, when tested at delays longer than the training delay, pigeons tend to choose the comparison associated with the short sample. The dominant explanation maintains that pigeons code durations in a retrospective, analogical form and that the choose-short effect arises because the analogical representation foreshortens during a delay interval (e.g., Grant, 1993; Grant \& Spetch, 1991, 1993; Grant et al., 1997; Roberts, Macuda, \& Brodbeck, 1995; Santi et al., 1993; Spetch, Grant, \& Kelly, 1996; Spetch \& Rusak, 1992b; Spetch \& Wilkie, 1982, 1983).

Recently, Santi, Ross, Coppa, and Coyle (1999) studied memory for empty time intervals in pigeons. In their procedure, the intervals to be timed were bounded by markers. On some trials, onset and termination of the empty interval to be timed, either 2 or $8 \mathrm{sec}$ in duration, was marked

This research was supported by Grant OGP 0443 from the Natural Sciences and Engineering Research Council of Canada. Correspondence concerning this article should be addressed to D. S. Grant, Department of Psychology, University of Alberta, Edmonton, AB, T6G 2E9 Canada (e-mail: douglas.grant@ualberta.ca). by 500 -msec light presentations, and on other trials was marked by 500 -msec tone presentations. Following acquisition with a 0 -sec delay, pigeons were tested with delays of $0,1,3$, and 9 sec. Regardless of whether the empty intervals were marked by tone or light, pigeons demonstrated a modest choose-short effect at the 1-sec delay and a choose-long effect at both the 3-sec delay and, especially, the 9-sec delay. Hence, in contrast to the typical result obtained when filled intervals are employed, Santi et al.'s (1999) empty-intervals procedure resulted in a tendency to choose the comparison associated with the long, rather than with the short, sample at delays longer than the training delay.

Although Santi et al.'s (1999) finding of a choose-long, rather than a choose-short, effect at longer delays suggests the intriguing possibility that memory processing may differ as a function of whether intervals to be timed are filled or empty, Santi et al. (1999) argued instead that their findings reflected timing of a new interval initiated by the second time marker on some trials. On such occasions, they argued, pigeons should tend to respond to the short-associated comparison at a 1-sec delay because the remembered duration, $1 \mathrm{sec}$, is closer to short $(2 \mathrm{sec})$ than to long $(8 \mathrm{sec})$. In contrast, on occasions when the second marker initiated timing a new interval and the delay was $9 \mathrm{sec}$, pigeons should tend to respond to the long-associated comparison because the remembered duration, $9 \mathrm{sec}$, is closer to long $(8 \mathrm{sec})$ than to short $(2 \mathrm{sec})$. The notion that the second marker might initiate timing of a new interval on some occasions is particularly plausible because the first and the second markers were the same physical stimulus.

The experiments reported in this article also assessed memory for empty time intervals in pigeons. In contrast to the procedure employed by Santi et al. (1999), in which the first (start) and second (stop) markers were always the same stimulus on any particular trial, the first and the second markers were always different colored keylights on any particular trial in the present experiments. In the consistent 
group, the first marker was a 1-sec presentation of three red keylights, and the second marker was a 1-sec presentation of three green keylights. In the inconsistent group, although the first and the second markers were always different on a trial, red and green served equally often as the first and second markers across trials. Hence, for subjects in the consistent group, the second marker should be unlikely to initiate timing of a new interval, because its role as a stop signal should be firmly established by the training regimen. Moreover, use of different start and stop markers in group consistent allowed empirical assessment of whether or not the second marker sometimes initiated timing a new interval, and that assessment is reported in Experiment 3.

\section{EXPERIMENT 1}

Pigeons were trained to respond to a line of one orientation after a short (2-sec) empty interval and to the alternative line orientation after a long (8-sec) empty interval. In group consistent, the onset of an empty interval was marked by presentation of red keylights, and the termination of the interval was marked by green keylights. In group inconsistent, red and green served as start and stop signals equally often across trials, although the onset and termination of an empty interval was marked by different colored keylights on any particular trial. Using consistent start and stop markers in group consistent was designed to reduce the likelihood that the second marker (i.e., green keylights) would, on some occasions, initiate timing a new interval.

As a second procedural modification of Santi et al.'s (1999) protocol, training trials involved a variable delay in the range of $1-3 \mathrm{sec}$. The purpose of this aspect of procedure was to reduce the likelihood that onset of the comparisons, rather than onset of the second marker, would terminate timing. Grant and Kelly (1996) have shown that use of short variable delays does not alter memory processing of filled intervals in pigeons. Hence, it is reasonable to assume that use of short variable delays in the present procedure would not qualitatively alter processing and that its effect would be limited to enhancing control over timing termination by the second marker.

Following acquisition, pigeons received consecutive retention tests in which the delays were $0.5,2( \pm 1)$, and $10 \mathrm{sec}$ in the first test and were $2( \pm 1), 10$, and $20 \mathrm{sec}$ in the second test. Because the procedure in group inconsistent was similar to that employed by Santi et al. (1999), a replication of their result (i.e., a choose-long effect at delays longer than the training delay) was anticipated. Interest focused on whether a similar choose-long effect would also occur in group consistent. The latter result would be consistent with the idea that pigeons process empty and filled time intervals differently.

Pigeons were also tested on occasional probe trials in which no sample (and, hence, no marker) was presented. (Although this test was conducted following Experiment 3 , it is reported here for clarity and economy of ex- position.) Studies employing filled temporal intervals have revealed that pigeons demonstrating a choose-short effect respond on no-sample trials as if the sample had been short (e.g., Fetterman \& MacEwen, 1989; Gaitan \& Wixted, 2000; Spetch \& Wilkie, 1983). Given the results of Santi et al. (1999), it seemed unlikely that birds would treat no sample as a short sample, at least in group inconsistent, in which a choose-long effect was anticipated. Alternatively, it could be argued that the choose-long effect arises following training with empty temporal intervals because pigeons code only the occurrence of a short sample and respond to the long-associated comparison by default, in the absence of a code indicating that a short sample had been presented. If so, pigeons demonstrating a choose-long bias during retention testing should demonstrate a similar bias on no-sample trials.

\section{Method}

\section{Subjects}

Sixteen Silver King pigeons (Columba livia), each approximately 1.5 years old at the start of training, were maintained at $80 \%$ of their free-feeding weight. The birds were housed individually in wiremesh cages and were given unrestricted access to water and health grit. Home cages were kept in a colony room, illuminated on a 12:12-h light:dark cycle, with light onset at $0600 \mathrm{~h}$. All the birds had prior experience in identity delayed matching-to-sample tasks using vertical and horizontal lines as sample and comparison stimuli. Eight birds were assigned at random to group consistent, and the remaining 8 were assigned to group inconsistent.

\section{Apparatus}

Training and testing was conducted in eight identical operant chambers, each measuring $29.0 \times 29.0 \times 24.0 \mathrm{~cm}$ (height $\times$ length $\times$ width). In each chamber, a horizontal alignment of three circular pecking keys (each $2.5 \mathrm{~cm}$ in diameter, and with side keys separated edge-to-edge from the center key by $3.0 \mathrm{~cm}$ ) was centered along one end wall. The key alignment was raised $22.5 \mathrm{~cm}$ from the barredfloor base of the chamber. Affixed behind each key was an Industrial Electronics, Inc. (Van Nuys, CA) in-line projector. All three projectors were capable of transilluminating red or green fields. Both sidekey projectors were capable of transilluminating a $0.7-\mathrm{cm}$-wide white line oriented $45^{\circ}$ to the left of vertical or $45^{\circ}$ to the right of vertical. A force greater than $0.15 \mathrm{~N}$ applied to any key was recorded as a keypeck. The top edge of a 5.0-cm-wide $\times 5.5-\mathrm{cm}-$ high rectangular opening was located $9.0 \mathrm{~cm}$ directly beneath the edge of the middle key. This opening provided access to a retractable food magazine. A 28-V lamp, recessed within the magazine opening, was activated when the food magazine was raised. Each chamber was enclosed in a sound- and light-attenuating booth. Within each booth, an exhaust fan provided ventilation and, supplemented by an external white noise generator, provided masking auditory stimulation. All the experimental booths were isolated in the same darkened running room. The only illumination inside the chamber was provided by activation of keylights and the magazine light. Experimental events were controlled from, and responses were recorded by, a microcomputer located in an adjoining room. Experimental sessions were conducted daily 6 days per week and began at approximately the same time each day.

\section{Procedure}

Training. For birds in group consistent, each trial began with a 1sec presentation of red light on all three pecking keys (start marker). Termination of the red lights was followed by a dark interval of either 2- (short) or 8-sec (long) duration. The end of the interval was 
marked by onset of green light on all three pecking keys that lasted for $1 \mathrm{sec}$ (stop marker). Termination of the green keylights was followed by a short, variable delay that ranged from 1 to $3 \mathrm{sec}$ in 0.5 sec increments. Termination of the delay was followed immediately by presentation of the right-slanted line comparison stimulus on one side key and the left-slanted line comparison stimulus on the remaining side key. A single peck to either comparison stimulus terminated both. For 4 subjects, a peck to the left-slanted comparison stimulus after the short sample and a peck to the right-slanted comparison stimulus after the long sample were designated as correct matches and thus resulted in reinforcement, consisting of $2.5 \mathrm{sec}$ of magazine-illuminated access to mixed grain from the food hopper. Alternatively, a peck to the right-slanted line after the short sample and a peck to the left-slanted line after the long sample were designated as incorrect matches and thus resulted in nonreinforcement, consisting of $2.5 \mathrm{sec}$ of darkness without mixed grain. For the remaining 4 subjects, these contingencies were reversed. For birds in group inconsistent, trials were the same as in group consistent, except that red and green served equally often as the start and stop markers across trials. On any particular trial, the start and stop markers were always of a different color.

Sessions consisted of an equal number of short- and long-sample trials. Within each session, the side-key location of the correct comparison stimulus was varied randomly across trials, with the restriction that, over all trials with each sample, the correct comparison stimulus appeared equally often on the left and right keys.

Each trial concluded with the onset of a dark intertrial interval (ITI), the duration of which varied randomly in 5-sec increments within a range of $10-30 \mathrm{sec}(M=20 \mathrm{sec})$. Each session consisted of 64 trials. The pigeons were advanced to extended-delay testing when they reached a criterion of two consecutive four-session blocks in which overall accuracy equaled or exceeded $85 \%$. Pigeons that had not reached criterion by Block 52 (Sessions 205-208) were dropped from the experiment.

Extended-delay testing. Testing consisted of two consecutive test phases, each consisting of eight test sessions. Each test session was preceded by two baseline sessions identical to those in training. In both test phases, all parameters and procedures for test sessions were identical to those of training, except that the delay between termination of the second marker and onset of comparison stimuli varied among three values. In the first test phase, on a randomly determined $75 \%$ of the trials, the delay was equivalent to that of training and hence varied between 1 and $3 \mathrm{sec}$ in 0.5 -sec increments. On a randomly chosen half of the remaining $25 \%$ of trials, a 0.5 -sec delay was interpolated between sample termination and comparison stimuli onset. On the remaining half of these trials, a 10-sec delay occurred. In the second test phase, as in the first, on a randomly determined $75 \%$ of the trials, the delay was equivalent to that of training and, hence, varied between 1 and $3 \mathrm{sec}$ in 0.5 -sec increments. On a randomly chosen half of the remaining $25 \%$ of trials, a $10-\mathrm{sec}$ delay was interpolated between sample termination and comparison stimuli onset. On the remaining half of these trials, a 20-sec delay occurred. In both test phases, both samples occurred equally often before each of the three delays, and the position of the correct comparison stimulus was balanced within sample-type and delayinterval factors. Correct responses on extended-delay trials were reinforced in accord with the contingencies of training. Eight consecutive training sessions, identical to those described previously, were interpolated between the two phases of extended-delay testing.

No-sample testing. Following completion of Experiment 3, the birds received four test sessions that were identical to their baseline sessions, except for the addition of 8 no-sample trials (total of 72 trials per session). On these trials, no markers were presented, and the comparisons followed the ITI and variable-delay interval. One nosample trial was scheduled to occur in each 9-trial segment during test sessions; on 4 of these trials, the short-associated comparison was on the right key and the long-associated comparison on the left key, and on the remaining 4 trials, this arrangement was the reverse.
A response to either comparison was reinforced on all no-sample trials. Percentage of choice of the short-associated comparison on nosample trials was computed for each bird. Each test session was preceded by two baseline sessions identical to those described in training.

In all the experiments reported in this article, statistical analyses employed $p<.05$ to define the critical region for rejection of the null hypothesis.

\section{Results}

\section{Training}

In group consistent, 7 of 8 birds met criterion prior to the last block of training (Block 52, Sessions 205-208). For these 7 birds, the mean number of blocks required to reach criterion (not including the 2 criterion blocks) was 26.1 (range, 16-44). In group inconsistent, only 2 of 8 birds met criterion and required 11 and 17 blocks of training, respectively, to do so (counterbalancing of the comparison stimuli across samples was preserved). Birds not reaching criterion were assigned a score of 52. A $t$ test revealed that blocks to criterion in group consistent $(M=$ 29.4) did not differ from that in group inconsistent $[M=$ $42.5 ; t(14)=1.69$ ]. Nonetheless, the fact that 7 of 8 birds acquired in group consistent, whereas only 2 of 8 acquired in group inconsistent, suggests that a consistent relationship between stimulus color and marker type (i.e., start and stop) rendered the task more tractable.

\section{Extended-Delay Testing}

Results of the first phase of testing, in which the delays were $0.5,2 \pm 1$ (as in training), and $10 \mathrm{sec}$, are shown in Figure 1. The pattern of retention was highly similar in the two groups. Both groups demonstrated high accuracy on both short- and long-sample trials at the training delay and a robust choose-long effect at the 10-sec delay. At the 0.5sec delay, accuracy was slightly lower than at the training delay, although neither group demonstrated a chooseshort effect at this delay.

A group $\times$ sample duration $\times$ delay analysis of variance (ANOVA) revealed a significant effect of delay $[F(2,14)=201.25]$ and a significant sample duration $\times$ delay interaction $[F(2,14)=4.87]$ as the only significant terms. The significant sample duration $\times$ delay interaction confirms the reliability of the choose-long effect at the 10sec delay.

The results of the second phase of testing, in which the delays were $2 \pm 1,10$, and $20 \mathrm{sec}$ are shown in Figure 2. As in the first phase of testing, the birds were highly accurate at the training delay and demonstrated a robust chooselong effect at delays greater than the training delay. An ANOVA identical to that performed on the data from the first phase of delay testing again revealed a significant effect of delay $[F(2,14)=137.39]$ and a significant sample duration $\times$ delay interaction $[F(2,14)=5.70]$ as the only significant terms.

\section{No-Sample Testing}

Collapsed across the four sessions of testing, the percentage of choice of the short-associated comparison 
stimulus on no-sample trials was $54.3 \pm 6.3$ (mean \pm $S E M$, based on 224 observations) and $50.2 \pm 0.5$ (mean $\pm S E M$, based on 64 observations) in groups consistent and inconsistent, respectively. Hence, neither group demonstrated a bias for one comparison over the other on nosample trials.

\section{Discussion}

Acquisition of accurate matching with empty time intervals proceeded much more slowly in the present exper- iment than in those reported by Santi et al. (1999). For example, in Experiment 1 of Santi et al. (1999) in which a tone was used as the marker, the birds received 3,000 trials of training. In the present experiment, in contrast, 7 birds (6 in group inconsistent) failed to reach satisfactory levels of accuracy after over 13,000 trials of training. Moreover, the 9 birds that did meet criterion ( 7 in group consistent) required an average of over 6,000 trials to do so. Several procedural differences may have contributed to betweenexperiments differences in task difficulty, including (1) use
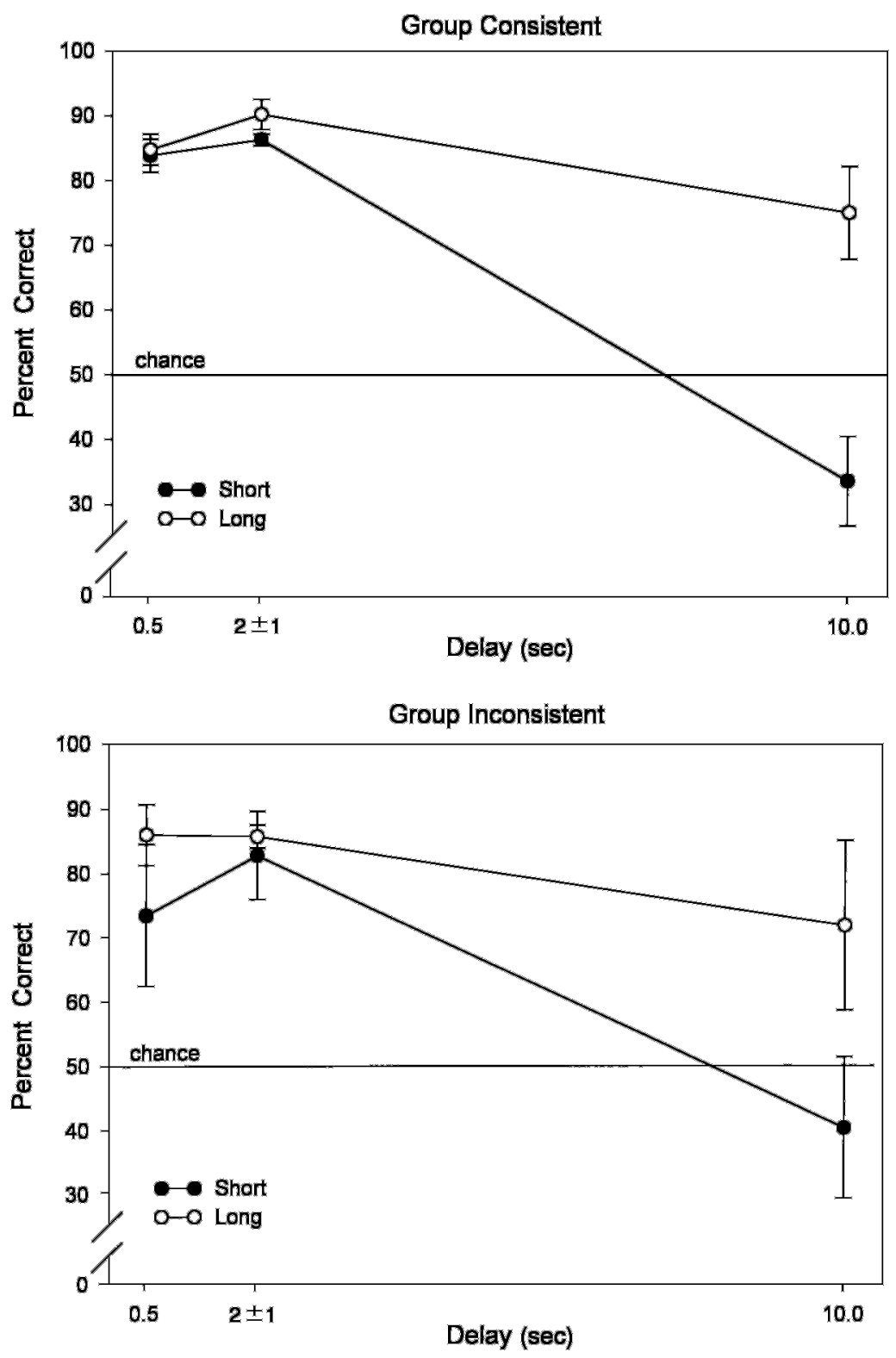

Figure 1. Percentage of correct responses as a function of delay on short- and longsample trials in group consistent and inconsistent in the first delay test in Experiment 1 . Each baseline $(2 \pm 1)$ datapoint is based on 1,344 and 384 observations in groups consistent and inconsistent, respectively. All other datapoints are each based on 244 and 64 observations in groups consistent and inconsistent, respectively. 
of highly discriminable color comparisons in Santi et al. (1999) versus use of less discriminable line comparisons in the present experiment, (2) use of a fixed, 0 -sec delay in Santi et al. (1999) versus use of a variable, nonzero delay in the present experiment, and (3) use of highly salient, ambient markers in Santi et al. (1999; tones and overhead lights) versus use of less salient, more localized keylights in the present experiment.

In accord with the results of Santi et al. (1999), both extended-delay tests revealed a robust choose-long effect at delays longer than the training delay. Moreover, the magnitude of the choose-long effect was statistically equivalent in the two groups. Santi et al.'s (1999) notion that the second marker sometimes initiates the timing of a new interval would explain the choose-long effect at longer delays in group inconsistent, in which the second (stop) marker on any particular trial often served as the first (start) marker on other trials. However, use of red lights as start markers and green lights as stop markers on all trials in group consistent was designed to reduce the likeli-
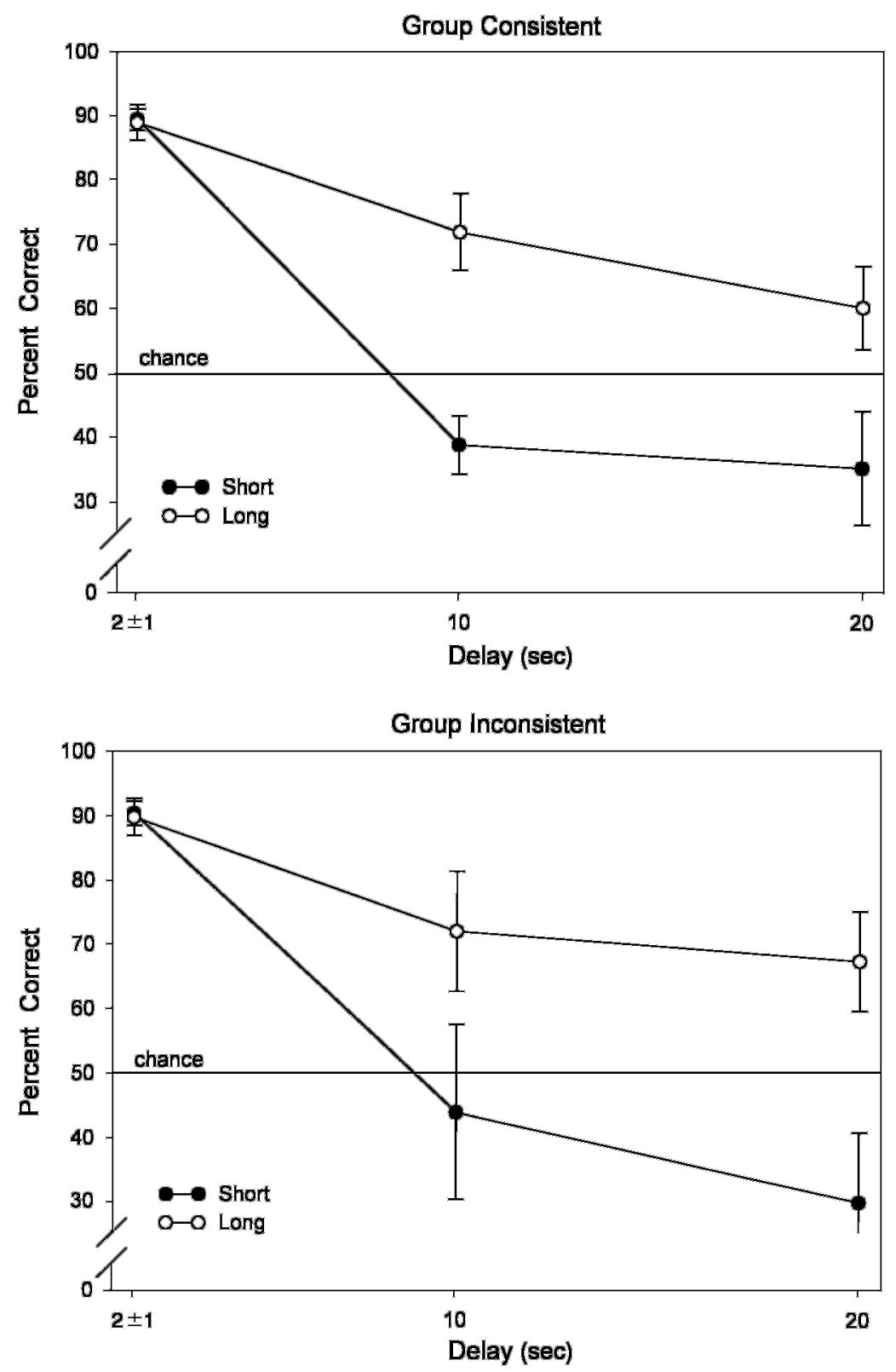

Figure 2. Percentage of correct responses as a function of delay on short- and longsample trials in group consistent and inconsistent in the second delay test in Experiment 1. Each baseline $(2 \pm 1)$ datapoint is based on 1,344 and 384 observations in groups consistent and inconsistent, respectively. All other datapoints are each based on 244 and 64 observations in groups consistent and inconsistent, respectively. 
hood that the second marker would induce the timing of a new interval. To the extent that the training regimen in group consistent was effective in preventing the second marker from initiating timing, the present results suggest that filled and empty intervals engage different forms of temporal coding. It could be argued, however, that the birds in group consistent were insensitive to the relation between color and type of marker and, therefore, that the second marker often initiated the timing of a new interval in this group. The next experiment examined whether the birds in group consistent were indeed sensitive to the relation between color and marker type, and Experiment 3 assessed directly the extent to which the second marker initiated timing in each group.

The finding that birds did not demonstrate a preference for the long-associated comparison on no-sample trials rules out one explanation of the choose-long effect during extended-delay testing. That explanation maintains that the choose-long effect arises from a strategy in which pigeons choose the long-associated comparison by default and choose the short-associated comparison only when memory for that event is present at the time of choice. That explanation, however, predicts a strong preference for the long-associated comparison and, hence, a very low percentage of short choices on no-sample trials, a result that was not obtained. Instead, it appears that absence of a time marker failed to engage the timing process and, therefore, pigeons responded randomly to the comparisons.

\section{EXPERIMENT 2}

Group consistent was exposed to test sessions in which, on half the trials, the relation between marker color and marker type was the same as that in previous training and testing (i.e., red start and green stop on same trials), whereas on the remaining half of the trials, this relation was opposite to that in previous training and testing (i.e., green start and red stop on opposite trials). To the extent that the birds in group consistent were sensitive to the relation between marker color and marker type, accuracy should be higher on same than on opposite trials. For comparison, trials in group inconsistent involving the marker sequence redgreen were designated same, and those involving the sequence green-red were designated opposite. Of course, for the birds in group inconsistent, these designations were arbitrary, in that their training and testing history involved an equal number of trials involving both sequences.

\section{Method}

\section{Subjects and Apparatus}

Those pigeons that had met the acquisition criterion in Experiment 1 ( $n=7$ in group consistent and $n=2$ in group inconsistent) served as subjects. The apparatus was the same as that in Experiment 1 .

\section{Procedure}

For the birds in both groups, test sessions were identical to the training sessions for group inconsistent in Experiment 1. That is, half the trials involved the marker sequence red-green (same as in the training for group consistent) and were designated same, and the other half involved the marker sequence green-red (opposite that in the training for group consistent) and were designated opposite. The birds in both groups received eight test sessions, each preceded by a single baseline session identical to those received in Experiment 1. The experiment was conducted immediately following the end of extended-delay testing in Experiment 1.

\section{Results}

Accuracy on same and opposite trial types as a function of sample duration, collapsed across the eight sessions of testing, is shown in Table 1. Accuracy was markedly lower on opposite than on same trials in group consistent, but was equivalent on the two types of trials in group inconsistent. A trial type $\times$ sample duration $\times$ group ANOVA revealed that the two groups were affected differently by the trial type manipulation, in that the trial type $\times$ group interaction was significant $[F(1,7)=12.15]$. To further assess the nature of this interaction, separate trial type $\times$ sample duration ANOVAs were performed on the data from each group. The ANOVA confirmed higher accuracy on same than on opposite trials in group consistent $[F(1,6)=$ 38.82]. Although the trial type manipulation appeared to have a greater effect on long- than on short-sample trials, that result was not statistically reliable, in that the trial type $\times$ sample duration interaction was not significant $[F(1,6)=$ 2.13]. A similar ANOVA performed on the data from group inconsistent failed to reveal any significant terms.

Although accuracy on opposite trials in group consistent exceeded chance $[t(6)=5.87]$, a session-by-session examination of performance revealed that accuracy on these trials increased across sessions, rising from 52.3\% on Session 1 to $73.7 \%$ on Session 8. A one-way ANOVA

Table 1

Percentage of Correct Responses (Mean $\pm S E M)$ on Same and Opposite Trials in Experiment 2

\begin{tabular}{|c|c|c|c|c|c|c|c|c|c|c|c|c|}
\hline \multirow[b]{3}{*}{ Group } & \multicolumn{6}{|c|}{ Same Trials } & \multicolumn{6}{|c|}{ Opposite Trials } \\
\hline & \multicolumn{2}{|c|}{ Short } & \multicolumn{2}{|c|}{ Long } & \multicolumn{2}{|c|}{ Mean } & \multicolumn{2}{|c|}{ Short } & \multicolumn{2}{|c|}{ Long } & \multicolumn{2}{|c|}{ Mean } \\
\hline & $M$ & $S E M$ & $M$ & $\overline{S E M}$ & $M$ & $\overline{S E M}$ & $\bar{M}$ & $\overline{S E M}$ & $\bar{M}$ & $\overline{S E M}$ & $M$ & $\overline{S E M}$ \\
\hline Consistent & 86.4 & 2.1 & 90.2 & 1.7 & 88.3 & 1.5 & 71.1 & 3.8 & 59.8 & 7.8 & 65.5 & 2.6 \\
\hline Inconsistent & 91.0 & 5.1 & 92.2 & 1.6 & 91.6 & 3.3 & 94.1 & 0.3 & 94.1 & 1.2 & 94.1 & 0.8 \\
\hline
\end{tabular}

Note-On same trials, the marker sequence was red-green, as it had been during training in group consistent. On opposite trials, the marker sequence was green-red, the reverse of that in training in group consistent. In group consistent, each cell entry (excluding the Mean column) is based on 896 observations. In group inconsistent, each cell entry (excluding the Mean column) is based on 256 observations. 
confirmed the increase in accuracy across sessions $[F(7,42)=7.06]$, and a $t$ test revealed that accuracy did not differ from chance in the first session $[t(6)=1.01]$.

\section{Discussion}

The subjects in group consistent demonstrated markedly lower accuracy on trials involving a marker-color-markertype relation different from that of their prior training history. Hence, the birds in group consistent were sensitive to that relation. The fact that accuracy on opposite trials in group consistent increased across testing sessions suggests that the birds in this group rapidly learned to report the duration of the empty interval that intervened between the "reversed" markers. Importantly, however, accuracy on opposite trials did not exceed chance on the first session of testing in group consistent. Hence, it is unlikely that the choose-long effect demonstrated in this group in Experiment 1 resulted from the second marker's initiating the timing of a new interval. Experiment 3 involved a direct test of whether the second marker on a trial sometimes initiated the timing of a new interval in either group inconsistent or group consistent.

\section{EXPERIMENT 3}

Santi et al. (1999, Experiment 3) reported data from trials on which the first time marker was omitted and the comparison stimuli were presented $0,1,3$, or $9 \mathrm{sec}$ after termination of the single time marker on that trial. A plot of percentage of long choices as a function of delay revealed V-shaped functions. Specifically, the percentage of long choices was particularly low at the 1-sec delay and was particularly high at the 9 -sec delay, relative to those at the 0 - and 3-sec delays. Certainly, these data indicate that presentation of the marker on single-marker trials did initiate timing of an interval (i.e., the delay interval). Santi et al. (1999) concluded that these data supported the further suggestion that the second marker on typical twomarker trials sometimes initiated the timing of a new interval. However, the latter conclusion appears unwarranted. Specifically, because the first and the second marker were physically identical, onset of the "second" marker on a first-marker-omitted trial would be completely indiscriminable from onset of the first marker on standard, twomarker trials. Hence, it is not difficult to understand why this "second" marker initiated timing of an interval. However, whether a second marker on a standard two-marker trial would also initiate timing of a new interval on some trials is not addressed by Santi et al.'s (1999) experiment. On a standard two-marker trial, the second marker is discriminable from the first, not because it is a different physical stimulus, but because it occurs shortly (i.e., either 2 or $8 \mathrm{sec}$ ) after a first marker.

In the present experiment, the first time marker was omitted on some trials. Thus, in group consistent, the first (red) marker was omitted, only the second (green) marker was presented, and comparison onset followed termination of the marker by either 0,2 , or $8 \mathrm{sec}$. To the extent that the
18 sessions of baseline training that intervened between Experiments 2 and 3 reestablished green as a marker to stop timing, little effect of delay length on the percentage of long choices would be expected. The subjects in group inconsistent served as a control. For these birds, onset of the "second" marker on first-marker-omitted trials should initiate timing of a new interval, if for no other reason than it was not possible to discriminate this marker from the first marker on typical two-marker trials. Hence, the birds in group inconsistent were expected to demonstrate a particularly low percentage of long choices when the comparisons appeared $2 \mathrm{sec}$ after the marker terminated and to show a particularly high percentage of long choices when the comparisons appeared $8 \mathrm{sec}$ after marker termination.

\section{Method}

\section{Subjects and Apparatus}

The subjects were the same as those in Experiment 2 ( $n=7$ in group consistent and $n=2$ in group inconsistent), and the apparatus was the same as that in Experiments 1 and 2.

\section{Procedure}

Eighteen baseline training sessions identical to those described in Experiment 1 intervened between the last session of testing in Experiment 2 and the first session of testing in the present experiment. The present experiment consisted of four test sessions, each preceded by two baseline sessions. For the subjects in both groups, test sessions consisted of 60 trials on which only the green time marker was presented for $1 \mathrm{sec}$. Recall that the green marker had served exclusively as the second (stop) marker in group consistent and had served equally often as the first and second marker in group inconsistent. Three equiprobable delays, 0,2 , and $8 \mathrm{sec}$, followed termination of the time marker, and the left-right positioning of the shortassociated and long-associated comparison stimuli was balanced within delay length. Choice of either comparison was reinforced during test sessions, and the percentage of long choices was recorded as a function of delay interval. All other aspects of procedure were the same as those in Experiment 1.

\section{Results}

The percentage of long choices as a function of delay following termination of the green time marker is shown in Figure 3. Group inconsistent, as was expected, revealed a V-shaped function, with a particularly low level of long choices at the 2-sec delay and a particularly high level of long choices at the 8-sec delay. In group consistent, on the other hand, there was little effect of delay on percentage of long choices.

A group $\times$ delay ANOVA revealed a significant interaction $[F(2,14)=20.54]$. To further analyze this interaction, one-way ANOVAs were performed on the data from each group. The ANOVA in group inconsistent revealed a significant effect of delay $[F(2,2)=24.32]$ and a significant quadratic trend $[F(1,1)=1372.37]$. In group consistent, in contrast, neither the effect of delay nor the linear or quadratic trend was significant.

\section{Discussion}

The data from group inconsistent are highly similar to those reported by Santi et al. (1999, Experiment 3). That 


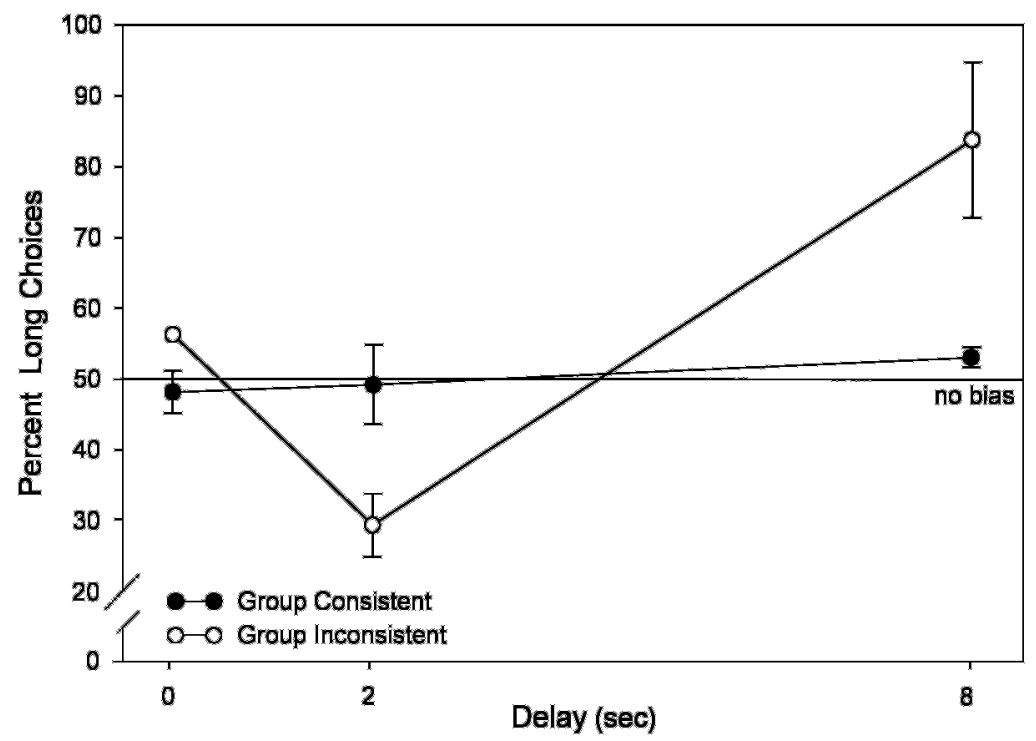

Figure 3. Percentage of choice of the long-associated comparison stimulus as a function of delay on trials in which only one marker was presented in groups consistent and inconsistent. The marker consisted of a 1 -sec presentation of three green keylights. This marker was the second (stop) marker on typical two-marker trials in group consistent. Each datapoint is based on 560 and 160 observations in groups consistent and inconsistent, respectively.

is, a V-shaped function arose when percentage of long choices was plotted as a function of delay. This pattern is precisely that expected if the time marker on single-marker trials initiated timing of a new interval. As was noted previously, however, this result does not necessarily imply that the second marker on standard two-marker trials sometimes initiates timing of a new interval.

Of primary interest in the present experiment were data from group consistent. In contrast to group inconsistent, choices in this group were not influenced by length of delay, suggesting that the green time marker, which had been experienced as the stop time marker in training, did not initiate timing of a new interval. Hence, the chooselong effect obtained in this group in Experiment 1 cannot be attributed to the second time marker's occasionally initiating the timing of a new interval. Moreover, given the equivalent magnitude of the choose-long effect in groups consistent and inconsistent, it appears unlikely that different processes are operating in the two groups. Indeed, it is more parsimonious to assume that whatever process(es) produced the choose-long effect in group consistent also operated to produce that effect in group inconsistent.

\section{GENERAL DISCUSSION}

The present experiments extended the research of Santi et al. (1999), in which pigeons were trained to time empty intervals of 2 or $8 \mathrm{sec}$ that were bounded by the presentation of a brief stimulus. In contrast with Santi et al's (1999) procedure, in which the same physical stimulus served as the first (start) and second (stop) time marker, the first and second markers on any particular trial were different colored keylights in the present research. In group consistent, 1 -sec red keylights always served as the first marker and 1sec green keylights always served as the second marker. In group inconsistent, red and green served equally often as first and second markers across trials.

In accord with Santi et al.'s (1999) results, and in contrast to the typical result when pigeons are trained with filled intervals, testing at delays considerably longer than the training value revealed a robust choose-long effect. The first experiment in the present series also examined performance on occasional probe trials in which no sample and, hence, no markers were presented. Although pigeons trained with filled intervals show a strong tendency to choose the short-associated comparison on no sample trials, the birds in both groups in the present study revealed indifference between the comparisons, choosing each on approximately half of the no-sample trials. In addition to revealing a second difference in performance when pigeons are trained and tested with filled versus empty intervals, that test also ruled out the possibility that pigeons choose the long-associated comparison by default and choose the short-associated comparison only when memory for a short interval is available at the time of choice.

Although Santi et al. (1999) attributed the choose-long effect to a tendency for the second time marker to initiate timing of a new interval (i.e., the delay interval) on some trials, Experiments 2 and, especially, 3 in the present series ruled out operation of such a mechanism, at least in group consistent. Experiment 2 revealed that birds in group consistent were sensitive to the fixed relation between marker 
type (start or stop) and marker color. Experiment 3 revealed that presenting only the second marker on probe trials did not initiate timing of a new interval in group consistent. Although presenting only the "second" marker did initiate timing on probe trials in group inconsistent, as was noted earlier in this article, this finding does not necessarily imply that presentation of the second marker on typical two-marker trials would also initiate timing a new interval. Moreover, given between-groups equivalence in the magnitude of the choose-long effect, it is most parsimonious to assume that whatever mechanism produced the choose-long effect in group consistent also operated to produce that effect in group inconsistent.

In summary, two empirical differences in performance have been identified as a function of whether pigeons are trained and tested with filled or empty intervals. First, pigeons demonstrate a choose-short effect at long delays when tested with filled intervals but demonstrate a chooselong effect at long delays when tested with empty intervals. Second, pigeons demonstrate a strong tendency to choose the short-associated comparison on no-sample trials in the filled-interval procedure but demonstrate no comparison-stimulus bias on such trials in the emptyinterval procedure. Although the research reported in this article revealed that the choose-long effect is not always a result of the second time marker initiating timing of a new interval, additional empirical work is required to identify why pigeons choose long at long delays and why they choose randomly on no-sample trials in the empty-interval procedure. Perhaps hybrid procedures (e.g., using start and stop markers in a filled-interval procedure) or transfer procedures (e.g., turning on the houselight during intervals after training in an empty-interval procedure) might reveal clues regarding mechanism.

\section{REFERENCES}

Fetterman, J. G., \& MacEwen, D. (1989). Short-term memory for responses: The "choose-small" effect. Journal of the Experimental Analysis of Behavior, 52, 311-324.

Gaitan, S. C., \& Wixted, J. T. (2000). The role of "nothing" in memory for event duration in pigeons. Animal Learning \& Behavior, 28, 147-161.

GRANT, D. S. (1993). Coding processes in pigeons. In T. R. Zentall (Ed.), Animal cognition: A tribute to Donald A. Riley (pp. 193-216). Hillsdale, NJ: Erlbaum.

GRANT, D. S., \& Kelly, R. (1996). The role of minimum wait time and sample discriminability in the coding of event duration in pigeons. Learning \& Motivation, 27, 243-259.

Grant, D. S., \& Kelly, R. (1998). The effect of variable-delay training on coding of event duration in pigeons. Learning \& Motivation, 29, 49-67.

Grant, D. S., \& SPETCh, M. L. (1991). Pigeons' memory for event duration: Differences between choice and successive matching tasks. Learning \& Motivation, 22, 180-199.

Grant, D. S., \& SPETCH, M. L. (1993). Analogical and nonanalogical coding of samples differing in duration in a choice-matching task in pigeons. Journal of Experimental Psychology: Animal Behavior Processes, 19, 15-25.

Grant, D. S., \& SPetch, M. L. (1994). The role of asymmetrical coding of duration samples in producing the choose-short effect in pigeons. Learning \& Motivation, 25, 413-430.

Grant, D. S., Spetch, M. L., \& Kelly, R. (1997). Pigeons' coding of event duration in delayed matching-to-sample. In C. M. Bradshaw \& E. Szabadi (Eds.), Time and behaviour: Psychological and neurobehavioural analyses (pp. 217-264). Amsterdam: Elsevier.

Kraemer, P. J., Mazmanian, D. S., \& Roberts, W. A. (1985). The choose-short effect in pigeon memory for stimulus duration: Subjective shortening versus coding models. Animal Learning \& Behavior, 13, 349-354.

Roberts, W. A., Macuda, T., \& Brodbeck, D. R. (1995). Memory for number of light flashes in the pigeon. Animal Learning \& Behavior, 23, 182-188.

Santi, A., Bridson, S., \& Ducharme, M. J. (1993). Memory codes for temporal and nontemporal samples in many-to-one matching by pigeons. Animal Learning \& Behavior, 21, 120-130.

SAnti, A., Ross, L., CopPa, R, \& Coy Le, J. (1999). Pigeons' memory for empty time intervals marked by visual or auditory stimuli. Animal Learning \& Behavior, 27, 190-205.

SPETch, M. L. (1987). Systematic errors in pigeons' memory for event duration: Interaction between training and test delay. Animal Learning \& Behavior, 15, 1-5.

Spetch, M. L., Grant, D. S., \& Kelly, R. (1996). Procedural determinants of coding processes in pigeons' memory for duration. Learning \& Motivation, 27, 179-199.

SPetch, M. L., \& RUSAK, B. (1992a). Temporal context effects in pigeons' memory for event duration. Learning \& Motivation, 23, 117-144.

Spetch, M. L., \& RusaK, B. (1992b). "Time present and time past.” In W. K. Honig \& J. G. Fetterman (Eds.), Cognitive aspects of stimulus control (pp. 47-67). Hillsdale, NJ: Erlbaum.

Spetch, M. L., \& Wilkie, D. M. (1982). A systematic bias in pigeons' memory for food and light durations. Behavior Analysis Letters, 2, 267-274.

Spetch, M. L., \& Wilkie, D. M. (1983). Subjective shortening: A model of pigeons' memory for event duration. Journal of Experimental Psychology: Animal Behavior Processes, 9, 14-30.

WILKIE, D. M. (1987). Stimulus intensity affects pigeons' timing behavior: Implications for an internal clock model. Animal Learning \& Behavior, 15, 35-39.

(Manuscript received March 12, 2001; revision accepted for publication July 12, 2001.) 